Revista Científica

https://revistacientifica.edu.pe

Febrero 2022

Volumen 1 / No. 5

ISSN: 2810-8108

https://revistacientifica.edu.pe

\title{
Modelo de gestión del conocimiento para medir el capital intelectual en la empresa hotelera Golden Green
}

Knowledge management model to measure intellectual capital in the hotel company Golden Green

Modelo de gestão do conhecimento para medir o capital intelectual na empresa hoteleira Golden Green

\section{ARTÍCULO GENERAL}

Yenny Milagritos Sifuentes Díaz

ysifuentes@unitru.edu.pe

https://orcid.org/0000-0001-9464-8294

Universidad Nacional de Trujillo
Cesar Alfredo Larios Franco

alarios@untels.edu.pe

https://orcid.org/0000-0002-4258-8549

Universidad Nacional Tecnológica de Lima Sur

Recibido 25 de Diciembre 2021 | Arbitrado y aceptado 25 de Diciembre 2021 | Publicado el 28 de Enero 2022

\section{RESUMEN}

El propósito del presente trabajo de investigación es elaborar un modelo de gestión del conocimiento para medir el Capital Intelectual en la empresa hotelera Golden Green. Para lo cual se plantea un modelo de medición de Capital Intelectual basado en cuatro etapas. En La primera etapa, se determina el análisis CATDWE donde se identifican los principales elementos para que la implementación del modelo sea viable. Luego, en la segunda etapa, se analiza la situación de una manera estructurada según la organización en particular. En la tercera etapa, se determina que el Capital Intelectual está constituido por tres dimensiones: Capital Humano $(\mathrm{CH})$, Capital Estructural (CE) y Capital Relacional (CR). Para cada dimensión se establece un conjunto de indicadores que resulta de la comparación de tres modelos de medición del Capital Intelectual: Modelo navegador Skandia, presentado por Edvinsson y Malone, modelo del Balanced Business Scorecard, desarrollado por Kaplan y Norton y el modelo Intelect de Euroforum. En la cuarta etapa, se considera un coeficiente directamente proporcional a cada dimensión planteando la siguiente ecuación. Capital Intelectual $=$ $\mathrm{x} \mathrm{CH}+\mathrm{y} \mathrm{CE}+\mathrm{z} \mathrm{CR}$.

Adicionalmente, se da también una valoración porcentual a cada indicador de las dimensiones del Capital Intelectual presentando una ecuación más detallada.

Se obtienen los valores de los coeficientes de las dimensiones del Capital Intelectual y de sus indicadores que son únicos y dependen de la organización de referencia. Estos coeficientes son calculados en base a un conjunto de encuestas a los elementos del análisis CATDWE. Al obtener los valores de los coeficientes se obtiene, finalmente, la ecuación para la medición del Capital Intelectual.

Palabras clave: Medición del Capital Intelectual, CATDWE, capital humano, capital estructural, capital relacional.

\section{ABSTRACT}

O objetivo deste trabalho de pesquisa é desenvolver um modelo de gestão do conhecimento para medir o Capital Intelectual na empresa hoteleira Golden Green. Para o qual é proposto um modelo de mensuração do Capital Intelectual baseado em quatro etapas. Na primeira etapa, é determinada a análise CATDWE onde são identificados os principais elementos para que a implementação do modelo seja viável. Então, na segunda etapa, a situação é analisada de forma estruturada de acordo com a organização em particular. Na terceira etapa, determina-se que o Capital Intelectual é composto por três dimensões: Capital Humano (CH), Capital Estrutural (CE) e Capital Relacional (CR). Para cada dimensão, é estabelecido um conjunto de indicadores que resulta da comparação de três modelos de medição do Capital Intelectual: modelo Skandia browser, apresentado por Edvinsson e Malone, modelo Balanced Business Scorecard, desenvolvido por Kaplan e Norton, e modelo Intelect do Euroforum.

$\mathrm{Na}$ quarta etapa, considera-se um coeficiente diretamente proporcional a cada dimensão, propondose a seguinte equação. Capital Intelectual $=\mathrm{x} \mathrm{CH}+\mathrm{y}$ $\mathrm{CE}+\mathrm{z}$ CR.

Adicionalmente, é também atribuída uma avaliação percentual a cada indicador das dimensões do Capital Intelectual apresentando uma equação mais detalhada. Obtêm-se os valores dos coeficientes das dimensões do Capital Intelectual e seus indicadores, que são únicos e dependem da organização de referência. Esses coeficientes são calculados com base em um conjunto de levantamentos aos elementos da análise CATDWE. Ao obter os valores dos coeficientes, obtém-se finalmente a equação para medir o Capital Intelectual.

Palavras-chave: Mensuração do Capital Intelectual, CATDWE, capital humano, capital estrutural, capital relacional.
RESUMO

O objetivo deste trabalho de pesquisa é desenvolver um modelo de gestão do conhecimento para medir o Capital Intelectual na empresa hoteleira Golden Green. Para o qual é proposto um modelo de mensuração do Capital Intelectual baseado em quatro etapas. Na primeira etapa, é determinada a análise CATDWE onde são identificados os principais elementos para que a implementação do modelo seja viável. Então, na segunda etapa, a situação é analisada de forma estruturada de acordo com a organização em particular. Na terceira etapa, determina-se que o Capital Intelectual é composto por três dimensões: Capital Humano $(\mathrm{CH})$, Capital Estrutural (CE) e Capital Relacional (CR). Para cada dimensão, é estabelecido um conjunto de indicadores que resulta da comparação de três modelos de medição do Capital Intelectual: modelo Skandia browser, apresentado por Edvinsson e Malone, modelo Balanced Business Scorecard, desenvolvido por Kaplan e Norton, e modelo Intelect do Euroforum.

$\mathrm{Na}$ quarta etapa, considera-se um coeficiente diretamente proporcional a cada dimensão, propondose a seguinte equação. Capital Intelectual $=x \mathrm{CH}+\mathrm{y}$ $\mathrm{CE}+\mathrm{z}$ CR.

Adicionalmente, é também atribuída uma avaliação percentual a cada indicador das dimensões do Capital Intelectual apresentando uma equação mais detalhada.

Obtêm-se os valores dos coeficientes das dimensões do Capital Intelectual e seus indicadores, que são únicos e dependem da organização de referência. Esses coeficientes são calculados com base em um conjunto de levantamentos aos elementos da análise CATDWE. Ao obter os valores dos coeficientes, obtém-se finalmente a equação para medir o Capital Intelectual.

Palavras-chave: Mensuração do Capital Intelectual,

CATDWE, capital humano, capital estrutural, capital relacional. 


\section{Introducción}

Es un gran reto para las empresas hoteleras mantener un buen nivel de satisfacción del cliente y posicionarse en un mercado cada vez más competitivo.

Las empresas hoteleras están formadas por activos tangibles como sus edificios, instalaciones, capital social, etc. y por activos intangibles como la satisfacción del personal, las competencias de las personas, el liderazgo, el trabajo en equipo, la capacidad de innovación, la cultura organizacional, la filosofía del negocio, la imagen de la empresa, la motivación, la estructura de la organización, el clima laboral, mecanismos de transmisión de conocimientos, etc. que es denominado como capital intelectual que genera ventajas competitivas y comparativas que les permitirán liderar en el mercado.

Con la finalidad de comprender la importancia del Capital Intelectual en el desarrollo empresarial presentamos los siguientes conceptos:

El capital intelectual es, a grandes rasgos, el conocimiento adquirido a través de la experiencia de una organización, institución o empresa. Se trata de una información intangible que permitirá a quien lo gestione adecuadamente aprovechar mejor las oportunidades, dando lugar a la generación de beneficios futuros.

Edvinsson fue uno de los primeros en ser consciente de la importancia del capital intelectual dentro de una empresa. Presenta el concepto mediante la siguiente metáfora: "una corporación es como un árbol. Hay una parte que es visible (las frutas) y una parte que está oculta (las raíces). Si solamente te preocupas por las frutas, el árbol puede morir. Para que el árbol crezca y continúe dando frutos, será necesario que las raíces estén sanas y nutridas. Esto es válido para las empresas: si sólo nos concentramos en los frutos (los resultados financieros) e ignoramos los valores escondidos, la compañía no subsistirá en el largo plazo".

De esta forma, este promotor del desarrollo del capital intelectual afirma que "gran parte del valor de una empresa es inexplicable e incontable. El conocimiento, la marca, innovación y otros activos invisibles concentran más creación de valor que los factores clásicos de producción en la gran mayoría de los negocios".

Steward, por su parte, lo define como "conocimiento, información, propiedad intelectual, experiencia, que puede utilizarse para crear valor. Es fuerza cerebral 
Yenny Milagritos Sifuentes Díaz, Alfredo César Larios Franco

colectiva. Es difícil de identificar y aún más de distribuir eficazmente. Pero quien lo encuentra y lo explota, triunfa". Steward afirma, además, que "en la nueva era, la riqueza es producto del conocimiento. Éste y la información se han convertido en las materias primas fundamentales de la economía y sus productos más importantes”.

De la misma forma, Skandia (compañía escandinava de seguros y servicios financieros), define el "capital intelectual como la posesión de conocimientos, experiencia aplicada, tecnología organizacional, relaciones con clientes y destrezas profesionales que dan a la empresa una ventaja competitiva en el mercado".

Según Alama (2010 p. 55) "El capital intelectual hace referencia al conjunto de activos intangibles que poseen las empresas y que conjuntamente con los activos tangibles forman parte de su patrimonio".

Villarreal y Villarreal (2017 p. 159) afirma que "El capital intelectual es el eje de las organizaciones en la era del conocimiento y la mentefactura, siendo el capital estratégico para establecer y mantener la ventaja competitiva. Una característica fundamental del capital intelectual, y que a la vez lo diferencia de otros tipos de capital, es que no se puede comprar o trasplantar de otro lugar; sino que se debe cultivar y desarrollar dentro de la organización".

Para Del Moral et al. (2007 p. 480) “A la hora de medir el valor de la institución, ya no sólo cuentan los activos tangibles, principalmente capital físico y financiero, sino que los activos intangibles, han adquirido gran importancia en su faceta de mantener a la misma en un entorno altamente competitivo y cambiante, convirtiéndose en el recurso por excelencia en las organizaciones."

Hoy en día las organizaciones más eficientes serán aquellas que sepan gestionar mejor sus recursos intangibles. Siempre se ha trabajado con el conocimiento de las personas, es cierto, pero ahora el reto es que ese conocimiento se constituya como un patrimonio de la organización, el cual no se le pueda arrebatar, dejando de ser un conocimiento informalmente asimilado y transmitido a la gestión formal del conocimiento, como activo de la organización mediante el desarrollo del capital intelectual que la conforma.

El Capital Intelectual como activo fundamental para el desarrollo eficiente de las organizaciones es necesario conocerlo y medirlo para en base a esas informaciones se tome decisiones para hacerlo crecer. La empresa hotelera Golden Green no dispone de 
Yenny Milagritos Sifuentes Díaz, Alfredo César Larios Franco

los recursos para el reconocimiento de estos valores intangibles, elementos invisibles y difíciles de cuantificar, y son los que están aportando hoy el porcentaje mayor del valor real que posee la empresa.

Por lo anterior, y reconociendo la importancia que posee la medición del capital intelectual para el crecimiento y desarrollo de las organizaciones que se enfrentan constantemente a un mercado competitivo la presente investigación está orientada a proponer un modelo de gestión del conocimiento para medir el capital intelectual lo que proporcionará información relevante para el proceso de toma de decisiones en la empresa hotelera Golden Green de Trujillo.

\section{Material y métodos}

\section{Objeto de estudio}

Población: La investigación tuvo como objeto de estudio a las variables para la medición del capital intelectual de la empresa hotelera Golden Green de Trujillo y lo conforma los dos socios de la empresa, seis empleados, veintidós huéspedes que se alojaron en la semana de observación y 4 proveedores principales.

Muestra: La muestra coincide con la población.

\section{Instrumentos recoleccion de datos}

La observación participante

La Entrevista.

La Encuesta

\section{Métodos y técnicas}

Tipo de estudio

Se realizó un estudio no experimental de corte transversal, debido a que no hubo manipulación de variables y la recolección de datos fue en un solo momento

\section{Metodología}

Para el desarrollo de este trabajo se revisó las definiciones más relevantes de Capital Intelectual en una línea de tiempo de los últimas tres décadas para luego generar la definición de Capital Intelectual para este estudio. 
Yenny Milagritos Sifuentes Díaz, Alfredo César Larios Franco

Luego se elaboró el modelo de Medición de Capital Intelectual para la empresa hotelera. Para que la aplicación del modelo de medición sea viable implementarlo debe reunir dos requisitos:

-Que sea factible realizarlo; es decir, debe tenerse los recursos disponibles para la implementación del modelo de medición de Capital Intelectual tales como, las personas para ejecutarlo, el presupuesto y el tiempo disponible.

-Que sea deseable; es decir; que los grupos humanos que intervienen tanto en la decisión de alto nivel estratégico como a las personas que se aplicarán los instrumentos de medición deben desear participar de este proceso.

Para que este modelo de medición sea viable implementarlo se siguió, en su elaboración, un conjunto de etapas en las que se consideran los paradigmas de los grupos humanos que conocen y viven la situación hotelera en mención.

Para la elaboración del modelo de medición de Capital Intelectual se planteó las siguientes etapas:

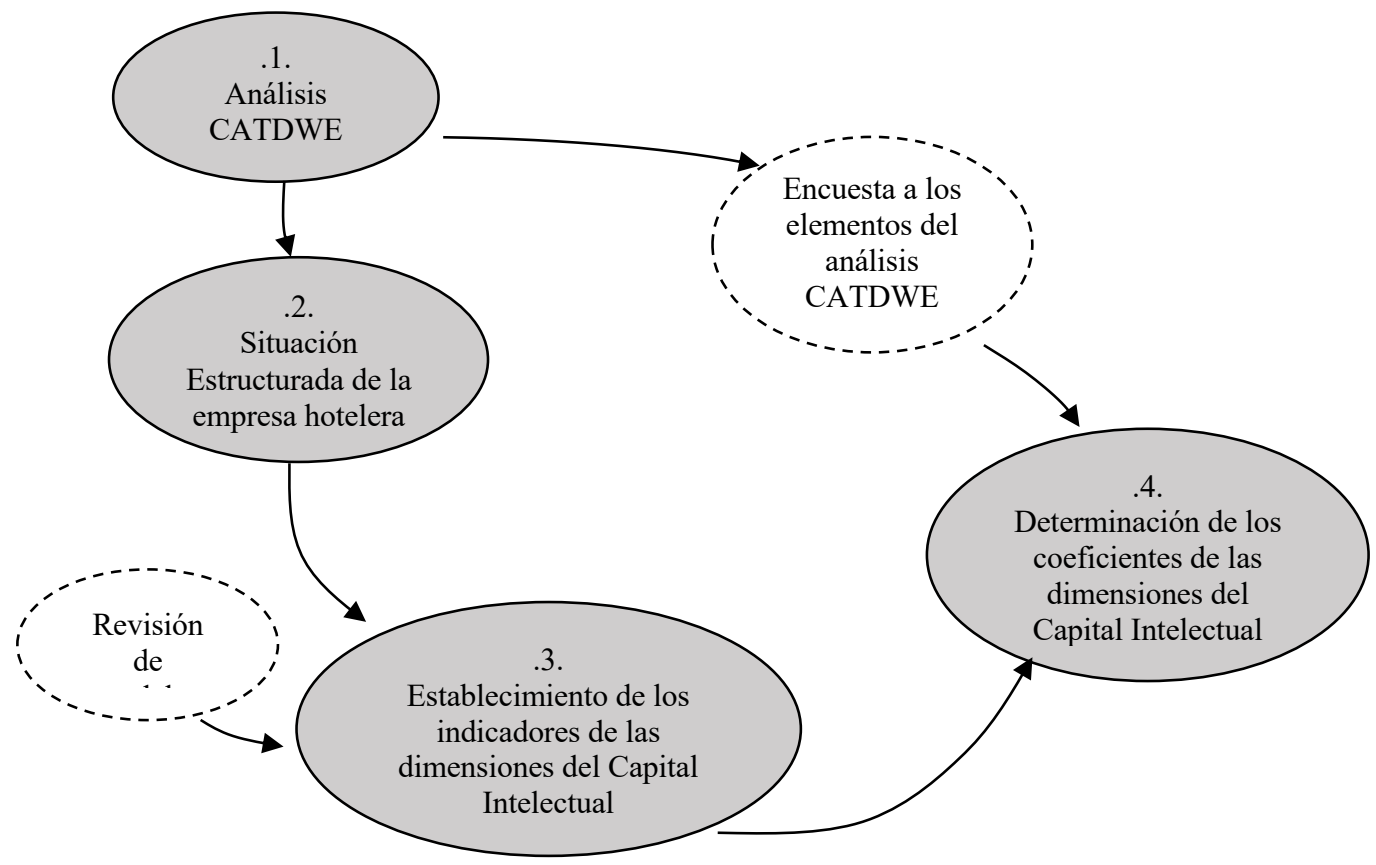

A continuación, describimos cada una de estas etapas:

\section{Etapa 1: Análisis CATDWE}

Esta etapa consiste en identificar seis elementos cruciales para que la medición del Capital Intelectual sea viable (factible y deseable) en la empresa hotelera propiciando un 
clima favorable para la implementación del modelo propuesto. Estos elementos son los siguientes:

a. Clientes (C): Son los beneficiarios o víctimas del proceso de transformación de medir el Capital Intelectual.

b. Actores (A): Son los que realizan el proceso de transformación de medir el Capital Intelectual, son a quienes vamos a aplicar los instrumentos de medición.

c. Transformación (T): Es el proceso de transformación. Para nuestro caso es la medición del Capital Intelectual.

No medir el Capital Intelectual

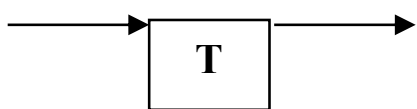

Medir el Capital Intelectual

d. Dueños (D): Son los dueños del proceso de transformación de medir el Capital Intelectual. Son los que permiten o no realizar el proceso de transformación.

Debe existir un compromiso e involucramiento de los dueños para empezar el trabajo.

e. Weltanschauung (W): Es la creencia que sustenta el proceso de transformación de medir el Capital Intelectual.

f. Entorno (E): Son las restricciones culturales, económicas, legales y tecnológicas que se tienen que tener en cuenta para realizar el proceso de medición del Capital Intelectual.

\section{Etapa 2: Situación Estructurada de la empresa hotelera}

La medición del Capital Intelectual debe ser un proceso único para cada organización el cual debe estar en función a su misión, visión, objetivos y estrategias, ya que los elementos que crean valor los determina cada organización.

\section{Etapa 3: Establecimiento de los indicadores de las dimensiones del Capital}

\section{$\underline{\text { Intelectual }}$}

Se determina los indicadores para cada dimensión del Capital Intelectual: humano, estructural y relacional en base al análisis y comparación de modelos de medición del capital intelectual. 


\section{Etapa 4: Determinación de los coeficientes de las dimensiones del Capital}

\section{Intelectual}

se considera un coeficiente directamente proporcional a cada dimensión planteando la siguiente relación del Capital Intelectual $=\alpha \mathrm{CH}+\beta \mathrm{CE}+\gamma \mathrm{CR}$.

Se obtienen los valores de los coeficientes $(\alpha, \beta, \gamma)$ que son únicos y dependen de la organización de referencia. Este coeficiente se calcula en base a un conjunto de encuestas aplicadas a los elementos del análisis CATDWE. Al obtener los valores de los coeficientes se obtiene la fórmula para la medición del Capital Intelectual.

\section{Resultados y discusión}

Primero, definiremos el capital Intelectual para el presente estudio para lo cual presentamos las definiciones de capital intelectual más relevantes de las últimas tres décadas.

\begin{tabular}{|l|c|l|}
\hline Edvinsson & 1996 & $\begin{array}{l}\text { Son las capacidades de perfeccionamiento que habitan en el interior } \\
\text { del ser humano, pero es algo más que el capital humano, pues } \\
\text { también incluye el capital estructural; son los conocimientos, } \\
\text { relaciones, know-how y otros intangibles de ese tipo. }\end{array}$ \\
\hline Bontis & 1996 & $\begin{array}{l}\text { La diferencia entre el valor de mercado de la empresa y el coste } \\
\text { reposición de sus activos. }\end{array}$ \\
\hline Brooking & 1997 & $\begin{array}{l}\text { Combinación de activos intangibles que permite funcionar a la } \\
\text { empresa }\end{array}$ \\
\hline Euroforum & 1998 & $\begin{array}{l}\text { Conjunto de activos intangibles de una organización que, pese a no } \\
\text { estar reflejados en los estados financieros tradicionales, en la } \\
\text { actualidad, el mercado percibe que generan valor o tienen potencial } \\
\text { de generarlo en el futuro. }\end{array}$ \\
\hline Stewart & 1998 & $\begin{array}{l}\text { Suma de todos los conocimientos que poseen todos los empleados } \\
\text { de una empresa y que le dan a esta una ventaja competitiva; se trata } \\
\text { de material intelectual (conocimiento, información, propiedad } \\
\text { intelectual, experiencia) que puede aprovechar para crear riqueza. }\end{array}$ \\
\hline Bueno & 2001 & $\begin{array}{l}\text { Es el conjunto de capacidades, destrezas y conocimientos de las } \\
\text { personas que generan valor, ya sea para las comunidades científicas, } \\
\text { las universidades, las organizaciones y la sociedad en general" }\end{array}$ \\
\hline
\end{tabular}

Fuente: elaboración propia, con base en Gonzalez y Rodriguez( (2016) y en Alama (2010)

Teniendo en cuenta las diferentes definiciones presentadas, definiremos el capital intelectual como el conjunto de recursos (conocimiento), capacidades y actividades a nivel interno y externo de la organización no tangibles que contribuyen a lograr el "para que" existe la organización. 
En base a esta definición se procedió a obtener el modelo de medición de capital intelectual de la empresa hotelera Golden Green desarrollando las cuatro etapas de la metodología descrita anteriormente

\section{Etapa 1: Análisis CATDWE}

Estos elementos son los siguientes:

\section{a. Clientes (C):}

En la empresa hotelera en mención, los clientes son los socios de la empresa hotelera, los empleados, los huéspedes y los proveedores ya que ellos se beneficiarían de un modelo de medición de capital intelectual.

b. Actores (A):

En la empresa hotelera Golden Green los actores son los socios de la empresa hotelera, los empleados, los huéspedes y los proveedores.

Los clientes y actores deben sentir la necesidad de medir el Capital Intelectual para ello debe realizarse charlas de concientización y motivación e informarles lo que se pretende con el modelo de medición del Capital Intelectual, sus objetivos, alcances, papel de cada cliente o actor y la utilidad de los resultados.

c. Transformación (T): Es el proceso de transformación. Para nuestro caso es la medición del Capital Intelectual.

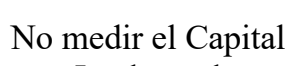

Intelectual

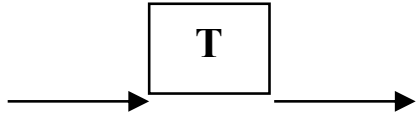

Medir el Capital Intelectual

d. Dueños (D): Para la organización donde estamos desarrollando el presente trabajo de investigación, los dueños son los socios de la empresa hotelera.

Debe existir un compromiso e involucramiento de los socios de la empresa hotelera para empezar el trabajo.

e. Weltanschauung (W): Es la creencia que sustenta el proceso de transformación de medir el Capital Intelectual.

La medición del Capital Intelectual permitirá conocer la estructura de los activos intangibles, lo que permitirá medirlos para mejorarlos y generar valor a la empresa hotelera. 
Yenny Milagritos Sifuentes Díaz, Alfredo César Larios Franco

f. Entorno (E): Son las restricciones culturales, económicas, legales y tecnológicas que se tienen que tener en cuenta para realizar el proceso de medición del Capital Intelectual.

\section{Etapa 2: Situación Estructurada de la empresa hotelera}

En esta etapa se presenta definida claramente la misión, visión y valores de la empresa hotelera Golden Green:

Misión:

Ofrecer al huésped el mejor servicio con un trato personalizado para hacerlos sentir como en casa, a través de colaboradores comprometidos.

Visión:

Mantenernos como empresa líder que ofrezca el mejor servicio de hospedaje, siempre comprometidos con el bienestar de los huéspedes

Valores:

Responsabilidad

Respeto

Orden

Trabajo en equipo

Honestidad

Proactividad

Innovación

Principios

Buen Servicio

Amor por el trabajo

Cordialidad

Etapa 3: Establecimiento de los indicadores de las dimensiones del Capital

\section{Intelectual}

El Capital Intelectual resulta de la integración de las siguientes dimensiones:

- Capital humano: son los valores y actitudes, aptitudes y capacidades que poseen los integrantes de la empresa 
Yenny Milagritos Sifuentes Díaz, Alfredo César Larios Franco

hotelera. El capital humano no es propiedad de la organización.

- Capital estructural: Es el medio por el cual el conocimiento de una empresa hotelera consigue explicitarse y formalizarse. Está integrado por las tecnologías, metodologías y procesos que hacen posible el funcionamiento de la organización. El capital estructural es propiedad de la organización, permanece en ella aun cuando su personal se retire. El capital estructural proporciona a la empresa hotelera mayor estabilidad que el capital humano.

- Capital relacional: Es la habilidad de las organizaciones, para adaptarse al entorno (clientes, proveedores, competidores, etc.) y sus variaciones, previéndolas y anticipándose a los cambios que en él se producen.

Para determinar los indicadores de cada una de las dimensiones del Capital Intelectual se realizó una investigación bibliográfica de tres modelos de medición del capital Intelectual. Los modelos investigados fueron:

1. Modelo navegador Skandia: sistema de capital intelectual. Presentado por Edvinsson y Malone en 1998. Muestra el aporte del Capital Intelectual dentro de la compañía, identificando la diferencia entre el capital financiero y el capital intelectual

2. Modelo del Balanced Business Scorecard. Desarrollado por Kaplan y Norton en 1992.Incluye indicadores de gestión no contenidos en los estados financieros convirtiéndose en una herramienta de apoyo a la toma de decisiones.

3. Modelo Intelect (Euroforum 1998).

Producto del análisis de estos tres modelos de medición del Capital Intelectual, se seleccionaron un conjunto de indicadores relacionados a una empresa hotelera. Luego, se realizó un cuadro para determinar a qué modelo pertenece cada uno de los indicadores elegidos. 
Yenny Milagritos Sifuentes Díaz, Alfredo César Larios Franco

\begin{tabular}{|c|c|c|c|c|}
\hline Dimensión & indicadores & $\begin{array}{l}\text { Navega } \\
\text { dor } \\
\text { Skandia }\end{array}$ & $\begin{array}{l}\text { Balanced } \\
\text { Business } \\
\text { Scorecard }\end{array}$ & Intelect \\
\hline \multirow{6}{*}{$\begin{array}{l}\text { Capital } \\
\text { humano }\end{array}$} & Satisfacción del empleado & $\mathrm{X}$ & $\mathrm{X}$ & $\mathrm{X}$ \\
\hline & Motivación & $\mathrm{X}$ & $\mathrm{X}$ & $\mathrm{X}$ \\
\hline & Liderazgo & $\mathrm{X}$ & & $\mathrm{X}$ \\
\hline & Capacidad de innovación & & $\mathrm{X}$ & $\mathrm{X}$ \\
\hline & Productividad & & $\mathrm{X}$ & $\mathrm{X}$ \\
\hline & Trabajo en equipo & & & $\mathrm{X}$ \\
\hline \multirow[b]{2}{*}{$\begin{array}{l}\text { Capital } \\
\text { estructural }\end{array}$} & Cultura organizacional & & $\mathrm{X}$ & $\mathrm{X}$ \\
\hline & $\begin{array}{l}\text { Huéspedes captados por } \\
\text { plataformas digitales }\end{array}$ & $\mathrm{X}$ & & $X$ \\
\hline \multirow{4}{*}{$\begin{array}{l}\text { Capital } \\
\text { relacional }\end{array}$} & Satisfacción del huésped & $\mathrm{X}$ & $\mathrm{X}$ & $\mathrm{X}$ \\
\hline & $\begin{array}{l}\text { Comunicación digital } \\
\text { (websites) }\end{array}$ & & $\mathrm{X}$ & $X$ \\
\hline & $\begin{array}{l}\text { Proporción de huéspedes que } \\
\text { repiten }\end{array}$ & $\mathrm{X}$ & $\mathrm{X}$ & $\mathrm{X}$ \\
\hline & Satisfacción de los proveedores & & & $\mathrm{X}$ \\
\hline
\end{tabular}

Etapa 4: Determinación de los coeficientes de las dimensiones del Capital

\section{Intelectual}

A cada dimensión del Capital Intelectual se le asignó un coeficiente (x, y, z) que es directamente proporcional a cada dimensión de la siguiente manera:

$$
\mathrm{Cl}=x \mathrm{CH}+y \mathrm{CE}+z \mathrm{CR}
$$

Donde:

$\mathrm{CI}=$ Capital Intelectual

$\mathrm{CH}=$ Capital Humano

$\mathrm{CE}=$ Capital Estructural

$\mathrm{CR}=$ Capital Relacional

$\mathrm{x}, \mathrm{y}, \mathrm{z}$ coeficiente de cada dimensión.

Para establecer el valor de cada coeficiente $(\mathrm{x}, \mathrm{y}, \mathrm{z})$ se realizó una encuesta a la muestra de estudio que constituyen los elementos del análisis CATDWE clientes, actores y dueños del proceso de transformación para que determinen el porcentaje de ponderación de cada coeficiente, obteniéndose los siguientes resultados: 


\begin{tabular}{|l|l|l|l|l|l|l|}
\hline $\begin{array}{l}\text { Dimensiones } \\
\begin{array}{l}\text { Capital } \\
\text { Intelectual }\end{array}\end{array}$ & $\begin{array}{l}\text { Coeficient } \\
\mathrm{e}\end{array}$ & $\begin{array}{l}\text { Socio } \\
\mathrm{s}\end{array}$ & $\begin{array}{l}\text { Empleado } \\
\mathrm{s}\end{array}$ & Huéspedes & proveedores & $\begin{array}{l}\text { Promedio } \\
\text { Coeficient } \\
\mathrm{e}\end{array}$ \\
\hline $\begin{array}{l}\text { Capital Humano } \\
(\mathrm{CH})\end{array}$ & $\mathrm{x}$ & 25 & 29.2 & 26.8 & 27.5 & 27.1 \\
\hline $\begin{array}{l}\text { Capital } \\
\text { Estructural (CE) }\end{array}$ & $\mathrm{y}$ & 30 & 27.5 & 27.3 & 30 & 28.7 \\
\hline $\begin{array}{l}\text { Capital } \\
\text { Relacional (CR) }\end{array}$ & $\mathrm{z}$ & 45 & 43.3 & 45.9 & 42.5 & 44.2 \\
\hline
\end{tabular}

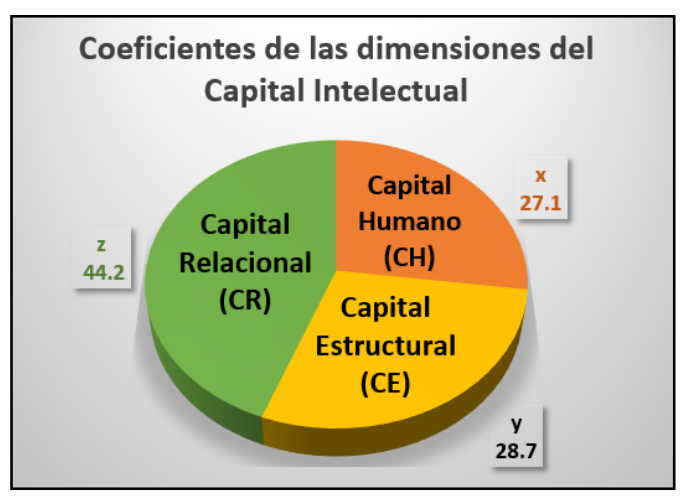

Teniendo los resultados de los coeficientes de la dimensión del Capital Intelectual, la ecuación para la medición del capital intelectual para la empresa hotelera de estudio es la siguiente:

$$
\mathbf{C l}=0.271 *(\mathrm{CH})+0.287 *(\mathrm{CE})+0.442 *(\mathrm{CR})
$$

Adicionalmente, a los indicadores de cada dimensión del Capital Intelectual se le asignó un sub coeficiente de la siguiente manera:

\begin{tabular}{|c|c|c|}
\hline Dimensiones CI & indicadores & Sub coeficiente \\
\hline \multirow{6}{*}{$\begin{array}{l}\text { Capital Humano } \\
(\mathrm{CH})\end{array}$} & Satisfacción del empleado (SE) & $\mathrm{x} 1$ \\
\hline & Motivación (M) & $\mathrm{x} 2$ \\
\hline & Liderazgo (L) & $\mathrm{x} 3$ \\
\hline & Capacidad de innovación (IN) & $\mathrm{x} 4$ \\
\hline & Productividad $(\mathrm{P})$ & $\mathrm{x} 5$ \\
\hline & Trabajo en equipo (TE) & $\mathrm{x} 6$ \\
\hline \multirow{2}{*}{$\begin{array}{l}\text { Capital } \\
\text { Estructural (CE) }\end{array}$} & Cultura organizacional $(\mathrm{CO})$ & $\mathrm{y} 1$ \\
\hline & $\begin{array}{l}\text { Huéspedes captados por plataformas } \\
\text { digitales (HD) }\end{array}$ & y2 \\
\hline \multirow{4}{*}{$\begin{array}{l}\text { Capital } \\
\text { Relacional (CR) }\end{array}$} & Satisfacción del huésped (SH) & $\mathrm{z} 1$ \\
\hline & Comunicación digital (websites) (CD) & $\mathrm{z} 2$ \\
\hline & $\begin{array}{l}\text { Proporción de huéspedes que repiten } \\
\text { (HR) }\end{array}$ & $\mathrm{z} 3$ \\
\hline & Satisfacción de los proveedores (SP) & $\mathrm{z} 4$ \\
\hline
\end{tabular}


Yenny Milagritos Sifuentes Díaz, Alfredo César Larios Franco

Siendo la ecuación detallada de medición del Capital Intelectual de la empresa hotelera de estudio la siguiente:

$$
\begin{aligned}
\mathbf{C l}= & 0.271 *\left(x_{1} S E+x_{2} L+x_{3} M+x_{4} I N+x_{5} P+x_{6} T E\right)+ \\
& 0.287 *\left(y_{1} C O+y_{2} H D\right)+ \\
& 0.442 *\left(z_{1} S H+z_{2} C D+z_{3} H R+z_{4} S P\right)
\end{aligned}
$$

Donde:

$\mathrm{CI}=$ Capital Intelectual

$\mathrm{SE}=$ Satisfacción del Empleado

$\mathrm{L}=$ Liderazgo

M= Motivación

$\mathrm{IN}=$ Capacidad de Innovación

$\mathrm{P}=$ Productividad

$\mathrm{TE}=$ Trabajo en Equipo

$\mathrm{CO}=$ Cultura Organizacional

$\mathrm{HD}=$ Huéspedes captados por Plataformas Digitales

SH= Satisfacción del Huésped

$\mathrm{CD}=$ Comunicación Digital

$\mathrm{HR}=$ Proporción de huéspedes que repiten

$\mathrm{SP}=$ Satisfacción de los proveedores

$\mathrm{x}_{\mathrm{i}}$ donde $\mathrm{i}=1 ; 2 ; 3 ; 4 ; 5 ; 6$ sub coeficientes de los indicadores del $\mathrm{CH}$

$y_{i}$ donde $\mathrm{i}=1 ; 2$ sub coeficientes de los indicadores del CE

$\mathrm{Z}_{\mathrm{i}}$ donde $\mathrm{i}=1 ; 2 ; 3 ; 4$ sub coeficientes de los indicadores del CR

Los indicadores de cada dimensión del Capital Intelectual también fueron ponderados por la muestra de estudio que constituyen los elementos del análisis CATDWE de nuestro proceso de transformación de no medir el Capital Intelectual a medir el Capital Intelectual. 
Yenny Milagritos Sifuentes Díaz, Alfredo César Larios Franco

La ponderación resulta de la importancia que le atribuyen los elementos del análisis CATDWE (2 socios de la empresa hotelera, a 6 empleados, a 22 huéspedes y 4 proveedores) a cada indicador valorando de la siguiente manera:

Para los indicadores del Capital Humano del 1 al 6; para los indicadores del Capital Estructural del 1 al 2 y para los indicadores del Capital relacional del 1 al 4. Considerando el 1 de mayor importancia.

Teniendo estas valoraciones se realizó la equivalencia porcentual, según los siguientes cuadros:

\begin{tabular}{|l|l|}
\hline \multicolumn{2}{|l|}{$\begin{array}{l}\text { Para los indicadores } \\
\text { del Capital Humano }\end{array}$} \\
\hline 1 & $30 \%$ \\
\hline 2 & $25 \%$ \\
\hline 3 & $15 \%$ \\
\hline 4 & $12 \%$ \\
\hline 5 & $10 \%$ \\
\hline 6 & $8 \%$ \\
\hline $\begin{array}{l}\text { Para los indicadores } \\
\text { del Capital Estructural }\end{array}$ \\
\hline 1 & $60 \%$ \\
\hline 2 & $40 \%$ \\
\hline
\end{tabular}

\begin{tabular}{|l|l|}
\hline \multicolumn{2}{|l|}{$\begin{array}{l}\text { Para los indicadores } \\
\text { del Capital Relacional }\end{array}$} \\
\hline 1 & $40 \%$ \\
\hline 2 & $30 \%$ \\
\hline 3 & $20 \%$ \\
\hline 4 & $10 \%$ \\
\hline
\end{tabular}

Se aplicó una encuesta a la muestra de estudio obteniendo los siguientes resultados: 


\begin{tabular}{|c|c|c|c|c|c|c|c|}
\hline $\begin{array}{c}\text { Dimensio } \\
\text { nes CI }\end{array}$ & indicadores & $\begin{array}{c}\text { Sub } \\
\text { coeficie } \\
\text { nte }\end{array}$ & $\begin{array}{l}\text { Soci } \\
\text { os }\end{array}$ & $\begin{array}{l}\text { Emple } \\
\text { ados }\end{array}$ & $\begin{array}{l}\text { Huésp } \\
\text { edes }\end{array}$ & $\begin{array}{l}\text { proveed } \\
\text { ores }\end{array}$ & $\begin{array}{c}\text { Promedio } \\
\text { porcentua } \\
1 \\
\end{array}$ \\
\hline \multirow{6}{*}{$\begin{array}{c}\text { Capital } \\
\text { Humano } \\
(\mathrm{CH})\end{array}$} & $\begin{array}{l}\text { Satisfacción del empleado } \\
\text { (SE) }\end{array}$ & $\mathrm{x} 1$ & 20 & 28.3 & 19.5 & 16.8 & 21 \\
\hline & Motivación (M) & $\mathrm{x} 2$ & 27.5 & 24.4 & 20 & 21.0 & 23 \\
\hline & Liderazgo (L) & $\mathrm{x} 3$ & 9 & 13.2 & 20.8 & 18.8 & 16 \\
\hline & $\begin{array}{l}\text { Capacidad de innovación } \\
\text { (IN) }\end{array}$ & $\mathrm{x} 4$ & 22.5 & 13.7 & 20.4 & 20.0 & 19 \\
\hline & Productividad $(\mathrm{P})$ & $\mathrm{x} 5$ & 12 & 10.2 & 10.6 & 15.4 & 12 \\
\hline & Trabajo en equipo (TE) & $\mathrm{x} 6$ & 9 & 10.2 & 8.7 & 8.0 & 9 \\
\hline \multirow{2}{*}{$\begin{array}{l}\text { Capital } \\
\text { Estructur } \\
\text { al (CE) }\end{array}$} & $\begin{array}{l}\text { Cultura organizacional } \\
\text { (CO) }\end{array}$ & y1 & 60 & 53.3 & 50.9 & 55 & 55 \\
\hline & $\begin{array}{l}\text { Huéspedes captados por } \\
\text { plataformas digitales (HD) }\end{array}$ & $\mathrm{y} 2$ & 40 & 46.7 & 49.1 & 45 & 45 \\
\hline \multirow{4}{*}{$\begin{array}{c}\text { Capital } \\
\text { Relaciona } \\
1 \text { (CR) }\end{array}$} & $\begin{array}{l}\text { Satisfacción del huésped } \\
\text { (SH) }\end{array}$ & $\mathrm{z} 1$ & 40 & 40 & 40 & 37.5 & 39 \\
\hline & $\begin{array}{l}\text { Comunicación digital } \\
\text { (websites) (CD) }\end{array}$ & $\mathrm{z} 2$ & 25 & 23.3 & 24.5 & 12.5 & 21 \\
\hline & $\begin{array}{l}\text { Proporción de huéspedes } \\
\text { que repiten (HR) }\end{array}$ & z3 & 25 & 26.7 & 25 & 17.5 & 24 \\
\hline & $\begin{array}{l}\text { Satisfacción de los } \\
\text { proveedores (SP) }\end{array}$ & z4 & 10 & 10 & 10.5 & 32.5 & 16 \\
\hline
\end{tabular}

Después de haber obtenido las valoraciones porcentuales de las dimensiones del Capital Intelectual y las valoraciones porcentuales de los indicadores de cada dimensión, se tiene la ecuación detallada del Capital Intelectual para la empresa hotelera del presente estudio:

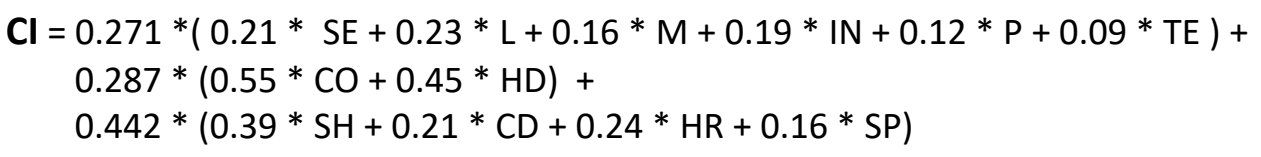

Donde:

$\mathrm{CI}=$ Capital Intelectual

$\mathrm{SE}=$ Satisfacción del Empleado

$\mathrm{L}=$ Liderazgo

$\mathrm{M}=$ Motivación

$\mathrm{IN}=$ Capacidad de Innovación

$\mathrm{P}=$ Productividad 
$\mathrm{TE}=$ Trabajo en Equipo

$\mathrm{CO}=$ Cultura Organizacional

$\mathrm{HD}=$ Huéspedes captados por Plataformas Digitales

$\mathrm{SH}=$ Satisfacción del Huésped

$\mathrm{CD}=$ Comunicación Digital

$\mathrm{HR}=$ Proporción de huéspedes que repiten

$\mathrm{SP}=$ Satisfacción de los proveedores

\section{Conclusiones}

Se determinó un modelo de gestión del conocimiento para medir el Capital Intelectual que se compone de cuatro etapas: Análisis CATDWE, Situación Estructurada de la empresa hotelera, Establecimiento de los indicadores de las dimensiones del Capital Intelectual y Determinación de los coeficientes de las dimensiones del Capital Intelectual

Se consideró que el Capital Intelectual estaba formado de tres dimensiones: Capital Humano, Capital Estructural y Capital Relacional.

Se determinaron 6 indicadores para el Capital Humano; dos indicadores para el Capital Estructural y cuatro indicadores para el Capital Relacional. Estos indicadores resultaron del análisis de los siguientes modelos de medición del Capital Intelectual: Modelo navegador Skandia, presentado por Edvinsson y Malone, modelo del Balanced Business Scorecard, desarrollado por Kaplan y Norton y el modelo Intelect de Euroforum.

Se consideraron coeficientes a las dimensiones del Capital Intelectual y subcoeficientes para sus indicadores; luego, se obtuvieron sus valoraciones a través de encuestas realizadas a los dos socios de la empresa, seis empleados, veintidós huéspedes que se alojaron en la semana de observación y 4 proveedores principales.

Finalmente, después de completar las cuatro etapas del modelo propuesto se formula la ecuación de medición del Capital Intelectual descrita en la página 12. 
Yenny Milagritos Sifuentes Díaz, Alfredo César Larios Franco

\section{Referencias Bibliográficas}

Alama, E. (2010). Capital Intelectual y ventaja competitiva. Editorial San Marcos.

Del Moral A., Pazos J., Rodríguez E., Rodriguez-Patón A. y Suárez S. (2007). Gestión del Conocimiento. Thomson Editores.

González J. y Rodríguez M. (2017). Gestión del conocimiento, capital intelectual e indicadores aplicados. Ediciones Diaz de Santos.

Villarreal, R, y Villarreal, T.(2017). IFA La Empresa Competitiva Sustentable en la Era del Capital Intelectual. Edición de Kindle. 